\title{
Addressing Human Factors in Burnout and the Delivery of Healthcare: Quality \& Safety Imperative of the Quadruple Aim
}

\author{
Michael R. Privitera
}

Medical Faculty and Clinician Wellness Program, University of Rochester Medical Center, Rochester, NY, USA

Email:Michael_Privitera@urmc.rochester.edu

How to cite this paper: Privitera, M.R. (2018) Addressing Human Factors in Burnout and the Delivery of Healthcare: Quality \& Safety Imperative of the Quadruple Aim. Health, 10, 629-644.

https://doi.org/10.4236/health.2018.105049

Received: March 30, 2018

Accepted: May 22, 2018

Published: May 25, 2018

Copyright $\odot 2018$ by author and Scientific Research Publishing Inc. This work is licensed under the Creative Commons Attribution International License (CC BY 4.0).

http://creativecommons.org/licenses/by/4.0/

\begin{abstract}
Human factors in the delivery of service are considered in many occupations of high impact on others such as airline industry and nuclear power industry, but not sufficiently in healthcare delivery. A common administrative framework of healthcare involves focus upon costs, quality and patient satisfaction (The Triple Aim). Many industries which support healthcare and healthcare administrators do not have firsthand knowledge of the complexities in delivering care. As a result, the experience and human factors of providing care are often overlooked at high level decision-making unless incorporated into the healthcare delivery framework, proposed as the fourth aim of The Quadruple Aim framework. Research is pointing to consequent negative effects on quality, safety, joy, meaning and sustainability of healthcare practice. High acute occupational stress and chronic occupational stress can cause direct and indirect effects on safety and quality of care. The biological, psychological and social consequences of burnout from excessive acute and chronic occupational stress are more of a threat to healthcare than commonly acknowledged. Patient safety, quality of care and clinician well-being are inextricably linked. This report will describe the process of transition from The Triple Aim to The Quadruple Aim administrative framework of healthcare delivery at the University of Rochester Medical Center. Developing the fourth aim of improving the experience of providing care, had high acceptability and aligned with other health system goals of optimization of safety, quality, and performance by applying a human factors/ergonomic (HFE) framework that considers human capabilities and human limitations. The goal of HFE is to fit the healthcare system to the human instead of the human to the healthcare system. Concepts include removal of extraneous cognitive load, using clinician neural resource (brain power) optimally for highest order decision mak-
\end{abstract}


ing in patient care. An integrative model of patient safety and clinician wellbeing is a product of this effort.

\section{Keywords}

Burnout, Human factors, Ergonomics, Safety, Clinician Wellbeing, Quadruple Aim, Integrative Model

\section{Introduction}

Many professions, especially in the delivery of healthcare, experience burnout. The human condition of burnout is the same among occupations, but the stressors differ by field.

Physician Burnout has increased from $45.5 \%$ in 2011 to $54.4 \%$ in 2014 [1]. Although clinician burnout has existed in medicine for decades, it had been considered solely the clinician's problem [2]. However, clinician burnout affects the individual, the organization, and the entire healthcare system through quality, safety, economic, legal risk as well as affecting availability to provide care and the experience by patients [3]-[8]. Patient healthcare satisfaction is also highly correlated with physician career satisfaction [9].

In our society there are accelerations of technological change proceeding on an exponential scale, which outpaces the human ability to adapt [10]. Connectivity has rapidly increased along with increased expectations and accountability. Widespread health professional burnout has been a predictable result. Across the nation, a "perfect storm" occurred of over-expectation of healthcare workers human capabilities and over-expectation of human capabilities by healthcare workers themselves groomed by their medical training culture.

The healthcare administrative framework of The Triple Aim [11] (lower costs, improve quality and improving patient satisfaction) has been extensively adopted, but misses human factors in the delivery of care that are now more important than ever. One author described a consequence as "phantom limb pain" manifested as clinician burnout since well-being of healthcare workforce was separated from system change initiatives in healthcare reform [12].

Two other landmark publications proposed a Quadruple Aim framework for healthcare which includes the Triple Aim objectives but includes a fourth aim described as: 1) Improving the work life of healthcare providers, including clinicians and staff [13], and 2) Creating conditions for the healthcare workforce to find joy and meaning in their work, and in doing so improving the experience of providing care [14].

Many industries which support healthcare and administrators who manage healthcare delivery do not have firsthand knowledge of the complexities of delivering care. As a result, the experience and human factors of providing care are often overlooked at high decision-making levels unless incorporated into the healthcare delivery framework, proposed as the fourth aim in The Quadruple 
Aim framework.

As more evidence of the negative impact of burnout emerges, this fourth aim has been more critical to patient safety, quality and the survival of the healthcare system than has previously been appreciated. The science of Human Factors/Ergonomics (HFE) has been applied to many other professions and more recently in the healthcare patient safety and quality of care arenas [15]. Their approach to humans and the systems they interface with includes awareness of human capabilities as well as human limitations [16]. HFE purpose is to optimize system performance while optimizing human well-being. Patient safety is one component of system performance. The HFE range includes physical, neurocognitive and organizational (macro) ergonomics and integrates contributions from biological, psychological and social sciences.

This manuscript will describe our experience at The University of Rochester Medical Center (URMC) on how using human factor/ergonomic approach to systemic improvements proved to be a highly acceptable and inter professionally understandable approach to align patient-centered and clinician-centered efforts to improve the experience of providing care.

\subsection{Cognitive Neuroscience in Care Delivery, Burnout and Errors}

Clinicians and administrators in healthcare have acknowledged that the number and complexity of many well-meaning quality and safety efforts in healthcare reform were overwhelming. These quality and safety efforts in healthcare came from legitimate authorities, but due to lack of interagency collaboration and harmonization, have been felt to be wearing out clinicians and backfiring in attempts to improve patient safety and quality by means of human factors unaccounted for in design. Finding a scientific framework to understand and communicate these dangers and improve the delivery of care experience became a goal of our institution. This approach is a-political, has immediate validity from application in other industries, and has a uniting effect toward better patient care. Neural resource (or brain power) is emerging as another resource that requires "budgeting" and optimum use in the delivery of healthcare.

Neural resource is the brain's energy resource needed for optimal brain functioning. It is a limited resource that needs to be replenished [17]. It is fundamentally used when we sift, sort, and classify, as well as using executive functions of the clinician brain required in healthcare delivery. Executive functions of the brain include focus, attention, self-control of behavior and speech, plan and organize, perspective taking, cognitive flexibility, medical and other decision making, ability to defer gratification, estimating time and working memory.

Neural resource gets used when we switch back and forth between different tasks (e.g., due to interruptions of our work process) or perform more than one task at a time-both frequent scenarios in the healthcare environment. Set-shifting (switching between tasks) and inter-task coordination (as required in multitasking) are executive function abilities, emerging from neural networks centered around the prefrontal cortex of the brain. 
Part of executive function is cognitive flexible memory (CFM) which allows individuals to draw upon facts and concepts from memory. CFM is used during medical decision-making that relies on access to facts from medical training to make a good differential diagnosis from the data of the patient's presentation. The anticipated next steps of being emotionally available to the patient and their family for conveying the plan or bad news are also controlled through CFM.

When neural resources are low due to acute excessive or chronic stress in burnout states, our brain shifts from behavior relying on executive functions and CFM to habit memory (HM), which is primarily controlled through the basal ganglia of the brain. In Human Error Theory literature, CFM function is roughly equivalent to "attentional (conscious)", while HM equals "automatic (unconscious)" processing [18] [19]. When the brain is in this survival mode, it "knows" it is working in a low neural resource condition, and automates response to a preceding stimuli without link to outcome that follows [20] [21]. This reduction in executive control compromises cognitive flexibility, medical decision-making quality, and degree of conscious choice as the clinician's behavior is more strongly guided by environmental signals than the individual's own goals and intentions. Hence, this condition is conducive to non-fund of knowledge errors. In this HM state, goal shielding occurs which shields against paying attention to anything else except the concrete goal of focus-a resource sparing action of HM function, which can contribute to premature closure error.

Burned out individuals have thinner prefrontal cortexes and enlarged amygdalae, which predict diminishment of executive function and poor modulation of emotions respectively [22] [23]. Individuals with burnout are more prone to attentional lapses and memory impairments [24].

Humans contribute to errors in two forms: Active and latent errors [25]. Active errors occur at the level of the frontline individual, and their effects are felt nearly immediately. Latent errors tend to be removed from the direct control of the individual and include poor design, installation and maintenance of equipment, management decisions, and organizational functioning. An unintended consequence from many well-meaning efforts to improve quality, reduce costs and improve patient satisfaction, when in the absence of consideration of human factors in the delivery of care, is increasing latent error risk. In other industries such as the airline industry, latent conditions are actively reduced and managed through industrial engineering and HFE principles to allow the best performance of the pilot. Currently in healthcare, attention to total workload and total expectations on the clinician does not exist. It has been proposed that creating a "manageable cockpit" (manageable expectations at the work site) for the clinician is a responsibility that must be shared by all levels and stakeholders of the healthcare system [26].

Total mental processing capacity is a limited function.

The Cognitive Load Theory posits three types of cognitive load:

1) Intrinsic load-inherent level of difficulty, thought to be immutable for the task such as cognitive load to reach diagnosis. 
2) Germane load-such as managing the emotional work of patient care, working with families.

3) Extraneous load-the burden in cognitive processing information that can be improved by better design [27] [28].

When extraneous cognitive load is too large, total cognitive load exceeds the limits of mental processing capacity, raising risk of error. Extraneous cognitive load is modifiable factor that can be the target of efforts to improve the experience of providing care and performance

\subsection{Work-Related Expectations during "Off" Time}

The need of clinician's brains to recover for optimum efficiency, has not been accounted for with technology advances that allows clinician connection to their work $24 / 7$ with the extended vigilance and accountability required. Job-related mandatory requirements that bleed into clinician off time needs to be rethought in a new human factor-based risk vs. benefit paradigm.

Currently healthcare settings do not grasp the down side risk of such extended vigilance. It is through culture and policy change that we can solidify the barrier between work and home. This barrier is required to allow for mental and physical recovery and retain a life outside of medicine, which pays off by better performance when at work in addressing the needs of patients, and contributing to career sustainability.

Two recent meta-analyses analyzed comparisons of individual and organizational interventions to reduce burnout [29] [30]. The strongest evidence for effectiveness was those organizational interventions to modify resources, working environment and work tasks to decrease stress.

\subsection{URMC Wellness Initiative for Quadruple Aim Framework}

Like other healthcare institutions, during the peak years of rapid roll-out of healthcare reform initiatives, faculty and staff at the University of Rochester Medical Center (URMC) experienced a very high level of occupational stress. Despite the human struggle to master each new initiative, multiple external authoritative forces required roll-out of each new initiative in a serial fashion but with little recovery time to get back up to speed and functionality. It was noticed by many, how healthcare workers were struggling to keep up with the changes, while attempting to still see patients and avoid errors.

\section{Methods/Approach for Burnout Reduction}

A hospital-based committee for Patient and Family Centered Care (PFCC Committee) has been active at our institution. The administrative topic focus began with improving patient satisfaction, then to disruptive behavior in clinicians, which led to the topic of reducing clinician burnout. Over an 18-month period, the PFCC Committee bench-marked with other institution's wellness efforts. We acknowledged that burnout was the driving and justifying force to build our wellness program, but that the term "wellness" may take on a more 
positively perpetuating effect. We also acknowledged that the wellness initiative would likely start off with individual based approaches, but later must also address organizational contributions to burnout. An official position of Medical Director of Medical Faculty and Clinician Wellness Program (MFCWP) to cover 0.2 FTE of physician time started in July 2015, in collaboration with existing resources such as employee assistance program, mental health services, occupational medicine, traditional wellness efforts, faculty development and informational technology efforts to improve provider satisfaction with the electronic medical record system.

The following relevant theories were incorporated into building a final wellness program: Business case for healthy workplace, conservation of energy, economy of scale, psychosocial safety climate, organizational health, emotional intelligence, cognitive load theory, preservation of clinician executive functions (affected by stress) to improve clinical decision-making, positive behavioral support (support intrinsic compassion, drive for quality of care, altruism, less coercion), neuro-cognitive ergonomic concepts for reducing cognitive strain in workflow and process designs, organizational ergonomics, bottom-up impact on work environment, clinician and staff engagement, and enhancing esprit de corps-(feeling of pride, fellowship, common loyalty shared by members of the medical center).

Application of concepts involved in the effects of stress on cognitive processes and decision making quality were helpful in aligning patient safety and quality efforts with staff wellbeing.

Strategic use of outside speakers was helpful to legitimize the internal efforts that were moving forward but accelerated after each speaker's visit.

Multiple Grand Rounds on clinician burnout and reduction occurred in 18 departments and divisions by request. Meeting individually with chairs, division chiefs, and other leaders allowed more input on unit/division/department-specific wellness needs.

Eleven wellness seminars were provided by volunteer faculty and staff each year. These seminars became a safe space to open up the topic of occupational stress and the toll that it takes. The process of the institution (Faculty Development Office) advertising seminars which had stress-validating titles, was a powerfully supportive intervention in and of itself. Examples of seminar titles:

- Overview of Burnout: Causes, Mechanisms and Reduction.

- Put Your Oxygen On First as You Take Care of Others.

- The Emotional Life of the Clinician.

- Finding Meaning in Medicine and Healthy Approaches to Clinician Stress.

An internal website was built to provide 24/7 access to resources of multiple video recordings made of the wellness seminars, some in shorter concise talks, literature to support initiating their own division support systems. Preventative-type coaching programs to assist with work/life balance are being made more available. 
The medical culture of endurance, isolation and silence is a barrier to get actionable input into improving operations. Many clinicians are painfully aware of the personal investment of time in training, debt, and family reliance upon them as well as the fear of being perceived as "weak" or a "fanatic". Gaining trust that there is an institutional commitment to action and wellbeing is key. Having anonymous methods of getting clinician input such as surveys, suggestion boxes, ombudsmen/clinician advocates needed to structurally be put in place. Once confidential input is obtained, the data can be talked about and processed openly in round table discussions with aggregate data since no source was identifiable.

One department operationalized a participatory management model referred to as Faculty Forum (FF) which was a model for other departments. FF did not set policy but served to bring forward issues, ideas, concerns, potential solutions, and whatever else is needed, to a variety of department venues which then were processed by senior leaders. Progress reports were made in general faculty meetings by the Faculty Forum Leader (FFL), who also had the role of faculty ombudsman. The FFL would bring forward faculty work/life issues of concern while preserving confidentiality of source.

A survey was conducted in three departments (Neurology, Medicine and Psychiatry) and with all Advanced Practice Providers (PAs and NPs) hospital wide. The results are included in this paper, with the approval of the URMC institutional review board. Within the survey, the two question version of the Maslach Burnout Inventory (MBI) [31] was used. These two items exhibit the highest factor loadings with their respective burnout domains and correlate strongly with the emotional exhaustion and depersonalization domains of burnout as measured by the full MBI. Permission was obtained from Mind Garden, Inc. for use of this proprietary scale [32]. The survey also requested 2 responses for each of the following questions: "Please tell us about the top two factors that most sustain your sense of meaning in your professional work.", "What are the top two factors that make your professional work most difficult?", "What practical suggestions do you have that may improve your workplace environment?".

A Wellness Strategic Planning Work Group was charged by senior leadership. The goals of the work group were:

1) Review data obtained from URMC survey responses, write-in suggestions, suggestions written on evaluations of the Program launch meeting, wellness seminar evaluations and existing literature.

2) Prioritize the projects/suggestions.

3) Propose projects, organizational structure and timeline for moving forward with the wellness initiative.

4) Synthesize proposals for presentation to senior leadership.

From 101 suggestions, the workgroup condensed these down to 22 ideas. Impact vs. Feasibility study was done by work group members assigning an Impact score and Feasibility score to each of the 22 ideas. Impact score of $1-4$ with 4 as highest presumes accomplishment, focus upon value and impact, breadth of applicability of improvement gained, degree of work stress reduction, degree of ef- 
ficiency, economic impact, job satisfaction, morale improvement. Feasibility score 1 - 4, with 4 as highest, ignores value or impact, focuses upon accomplishment, administrative support/ buy-in, expense, time frame, staffing factors, faculty/participant factors, institutional support/buy-in, etc. Voting Rules-voting on Impact levels: Up to 5 votes allowed for \#4 Impact level, Up to 5 votes allowed for \#3 Impact Level. Can vote as many \#2 Impact levels and \#1 Impact levels as they wanted. Voting on Feasibility Levels: No limits on number of times they can vote on any Feasibility level. Mean Impact score and Mean Feasibility score for each idea were added together to make an $I / F$ Index score for each idea. The higher the $I / F$ index, the more we would prioritize these interventions. The work group met monthly and voted on the most crucial matters pertaining to clinician wellness. Senior Leadership reviewed the work group report and has agreed with taking action on the findings and suggestions.

We have recently instituted measurement of well-being in residents and soon faculty, but results are not yet available.

\section{Results}

A three-Department and all Advanced Practice Providers (APPs) wellness survey had 528 respondents. Medicine $(n=202$, unable to accurately calculate response rate due to overlapping department list serves), Neurology (response rate: $87 / 157=55.4 \%$ ); Psychiatry (response rate: 71/94 $=75.6 \%$ ); APP (response rate: $173 / 550=31.5 \%$ ). $42 \%$ of $\mathrm{MD} / \mathrm{DOs}$ and $52 \%$ of APPs reported high burnout/emotional exhaustion. High burnout/emotional exhaustion was assessed if respondents chose either once a week, a few times a week, or every day as an answer to "I feel burned out from my work" In addition, 32\% of MD/DOs and $45 \%$ of APPs reported high callousness/depersonalization. High callousness/depersonalization was assessed if respondents chose either a few times a month, once a week, a few times a week, or every day as an answer to "I have become more callous toward people since I took this job" [31].

There was a dose-related effect of increasing burnout with increase $\%$ of their position (\% Full Time equivalent, or FTE) that is clinical work, compared to research, administration, or teaching. Linear trend for emotional exhaustion with \% clinical FTE was $\mathrm{p}<0.05$ and linear trend for callousness/depersonalization with $\%$ clinical FTE was $\mathrm{p}<0.0001$. Since the most frequently cited reason that sustains their sense of meaning was patient care itself (54\% of all respondents) a clear picture emerged that there are factors interfering with doing patient care. The interfering factors were likely wearing them down more in a dose-related fashion, the more clinical work exposure they have.

Our institution self-insures for staff healthcare. Using result-based projections from existing literature and burnout rates, we estimate that for every $\$ 1,000,000$ paid out on URMC clinicians' own medical claims, $\$ 243,000$ could be saved, if high burnout clinicians could be converted into low burn out clinicians [33]. For every $\$ 1,000,000$ in revenue from Patient Satisfaction scores from clinicians who work at URMC, $\$ 169,000$ more could be obtained if there was a conversion of 
High Burnout to Low Burnout clinicians [34].

Table 1 gives examples of quotations to document the evolution of the wellness initiative in a Quadruple Aim Framework at URMC. It shows progression from fear of raising the issue of burnout to clinician wellness becoming part of the institution strategic plan, 30 months later.

Four stages of intervention were designed from prioritization efforts and would build upon each other. This staging and roll out plan is outlined in the four year plan of getting to where we want to be: Decrease Burnout and improve Wellness. This plan commenced 24 months into the initiative and currently is in process (see Scheme 1).

\subsection{Integrative Model: Patient Safety and Staff Well-Being}

Much of attention in patient safety literature focuses upon setting up barriers to error as if layers of Swiss cheese. In unfortunate circumstances when the holes in these slices line up such that trajectory of error slips through all the slices (barriers), an incident occurs. In healthcare reform, quality and safety metrics, laws regulations and guardrails abounded and multiplied, but unfortunately without interagency collaboration or harmonization of mandates. Normal daily processes of healthcare delivery have paradoxically become thwarted, raising risk of error from acute extreme stress as well as constitutional changes in healthcare staff from chronic stress and burnout effects on their brain, hormones and excessive glutamate excretion [35] [36] [37].

As a result of this work, a new model was designed to include concepts from patient safety education, such as Reason's Swiss Cheese Model that promotes

Table 1. Selected quotes on wellness initiative roll out which began 7-1-15.

\section{Launch meeting of Wellness Program}

Senior Leader: "(Medical Director of Medical Faculty and Clinician Wellness Program) was brave to bring these things forward to initiate wellness efforts."

Audience participant: "We ought to consider why he had to be brave to bring these issues up in our medical culture."

\section{7 months into initiative:}

Visiting national speaker: "From all the places I have seen, this institution is ahead of the curve on improving focus on clinician wellbeing."

\section{8 months into initiative}

Senior Leader: "The difference here is palpable, there is a definite difference and impact of wellness efforts."

New faculty member: "The culture here is so attuned to wellness compared to where I came from. There has been a noticeable effect here by these efforts."

Senior Administrator: "Over and over in faculty retreats we hear how important clinician wellness is in providing care to patients."

30 months into initiative

National Academy of Medicine (NAM) Action Collaborative on Clinician Wellbeing website: URMC's NAM Statement on Commitment to Clinician Well-Being and Resilience:

"In recognition of the significant consequences of clinician burnout, the University of Rochester Medical Center (URMC) has made this issue a top priority by including it as a major pillar of our Strategic Plan... We embrace the Quadruple Aim, which places equal value on clinician job satisfaction with the other tenets of the IHI's Triple Aim... Human factor and ergonomic educational modules are being integrated into our quality and safety curriculum." 
Year 1

Medical Faculty and Clinician Wellness Program

Wellness Strategic Planning Work Group

Phase I = Clinicians

\section{1st Stage Project Focus}

1. Electronic Medical Record Provider

Satisfaction Campaign

2. Mobile team: Consultation and support to Chairs, Division Chiefs,

Center Heads.

3. Coaching (Preventative)

4. Continued Wellness seminars

5. Medical Staff Office Project

(requirement organization)

6. Leadership In-services on Burnout and

Human Factor-based Leadership

7. Provider wellness representation in all new and review of old clinical initiatives

8. Resident specific Issues with Clinical Learning Environment Review-CLER (Accreditation Council of Graduate

Medical Education- requirement)

9. Clinician Wellbeing Measurement and

understand relationship to quality, safety and other hospital metrics.
Year 2

Year 3

\begin{tabular}{|c|c|c|}
\hline $\begin{array}{l}\text { Phase II } \\
\text { Wellness Strategic } \\
\text { Planning Work Group } \\
\text { Clinicians } \\
\text { Additionally Includes: } \\
\text { Researchers } \\
\text { School of Nursing Faculty } \\
\text { Nursing Practice } \\
\text { Social Work } \\
\text { Chief Medical Officer } \\
\text { Occupational Medicine } \\
\text { Human Resources } \\
\text { Employee Assistance Program } \\
\text { Existing Wellness efforts for staff } \\
\text { Biometric Screening } \\
\text { Healthy Living experts } \\
\text { Quality and safety representatives } \\
\text { Subcommittees: } \\
\text { Phase I Clinicians } \\
\text { - Resident Wellness }\end{array}$ & $\begin{array}{l}3^{\text {rd }} \text { Stage Project Focus } \\
\text { 1. Wellness as quality indicator } \\
\text { 2. Zero Tolerance for disruptive } \\
\text { behaviors, but graduated spectrum } \\
\text { of interventions informed from } \\
\text { relationship between burnout and } \\
\text { disruptive behavior. } \\
\text { 3. Exosystem program development } \\
\text { (ways to reduce stress on } \\
\text { family life outside medicine) } \\
\text { 4.Examine Shadow Work (unseen, } \\
\text { unmeasured, unpaid jobs that fill } \\
\text { your day) } \\
\text { 5.Department level champions and } \\
\text { wellness efforts collaborative with } \\
\text { medical center wellness efforts. }\end{array}$ & \multirow[t]{3}{*}{$\begin{array}{l}\text { sing Faculty } \\
\text { sing }\end{array}$} \\
\hline & $\longrightarrow \longrightarrow \longrightarrow$ & \\
\hline \multicolumn{2}{|c|}{$\begin{array}{l}\text { 2nd } \text { Stage Project Focus } \\
\text { 1. Constituency input into workflow solutions } \\
\text { 2. Constituency input into Leadership ratings. } \\
\text { 3. Strategy Group for stronger clinician/administrator partnerships } \\
\text { 4. Integrate wellness efforts with disruptive behavior reduction } \\
\text { 5. Workflow and Scope of Practice committee } \\
\text { 6. Wellbeing measurement for other sectors } \\
\text { 7. Quadruple Aim Focus as Medical Center }\end{array}$} & \\
\hline
\end{tabular}

Year 1 of plan began two years after initial roll out of initiative.

Scheme 1. Getting to where we want to be: Decrease Burnout-Improve Wellness-4 year plan*.

defense barriers against incidents integrated with what we know about the impacts of burnout changing the cognitive abilities of the clinician, degrading the quality of work that follows. This integrative model visually conveys factors that drive healthcare operations and efforts at patient safety, but also gives a place for factors that thwart good care, though well-intended, both acutely as well as chronically. Organizational/systemic factors that affect the human clinician must be factored in a model that conveys usual care (the predominant amount of time) as well as the care with trajectory toward incident (the minority of time) to realistically balance risk versus benefits involved in proposed or existing patient safety and quality efforts (see Figure 1). Including safety concepts with well-being concepts in the same diagram helps integrate efforts of both. It more realistically conveys human capabilities as well as human limitations to synergize efforts instead of causing competing efforts by siloed efforts.

Discernment needs to occur on how strategic safety barriers are helpful in reducing error but also the downside risk of well-meaning but excessive or ineffective barriers, metrics, rules, policies, regulations that thwart good care of the patient [38] [39]. High acute stress affects risk of error in one way, but chronic stress effect on risk of error additionally occurs by anatomical and physiological changes that burnout inflicts on the clinician's brain with subsequent negative effects on quality of care provided. There is a sense of urgency that must be maintained to help reduce burnout.

\subsection{Time Line of Changes}

Time Line of external national influences and internal administrative and structural changes at URMC are outlined in Figure 2. 




*Latent Failures include poor design, installation, and maintenance of equipment, management decisions, and organizational functioning, and thwarted care leading to acute high stress and chronic high stress. Lower portion of figure adapted from: Taylor-Adams S, Vincent C. Systems Analysis of Clinical Incidents. The London Protocol. Mar 17, 2001 Clinical Safety Research Unit. Imperial College London.

Figure 1. Integrative model: Patient safety and staff wellbeing.

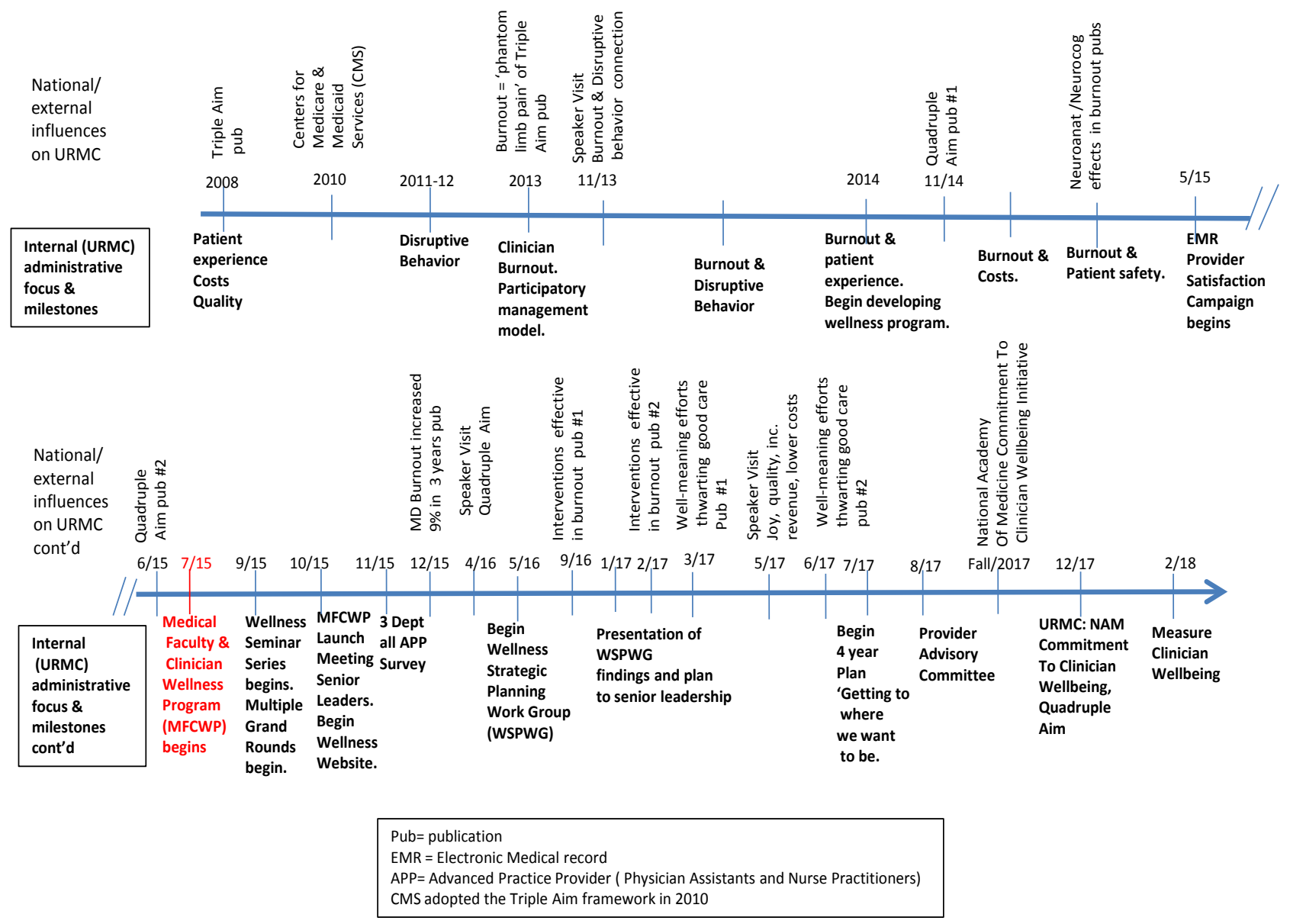

Figure 2. Time line: External and internal. 


\section{Discussion}

Leadership will require new skills and new processes to help their institutions and constituents adjust to expectations and changes of such technological accelerations and with the increasing expectations that occur. Reduction of burnout and improvement in clinician wellbeing is critical to patient safety, and is visually illustrated in Integrated Model for Patient Safety and Staff Wellbeing. Some healthcare interventions, though well-meaning may thwart good care, paradoxically decreasing safety and quality of care. Resource utilization and two-way communications need to be re-imagined in this context of helping the human adaptation process. Internal communication, feedback, and input back to leadership need more rapid methods. There is great need in these times to create stronger partnerships between clinicians and administrators [40] [41]. Improved provider well-being will lead to improved satisfaction, enhanced engagement and care relationships, greater compliance and efficiency, all resulting in expected better outcomes of care and sustainable practice of our healthcare providers [5] [8] [9] [42] [43]. Limitations of the study include the use of perceptual data, quotes and milestones. However overall improvements have been captured by doing so. In the future it will be useful to see how metrics of wellbeing will correlate with improved safety and quality, decreased costs, and improved patient satisfaction scores over some years.

\section{Strategic Direction}

- Organizational awareness of burnout and need for Quadruple Aim Approach as part of the institutions' strategic plan.

- Clinician awareness of burnout and resources need to be available to help their individual improvement.

- Leadership listening and commitment to action.

- Improve clinician/administrator relationships.

- Human factor based leadership training to help leaders understand burnout, causes, and mechanisms and mitigating interventions that can be applied in multiple situations. These efforts work well with Lean principles in improving workflows that are clinician-centric.

- Build in organizational structures that promote clinician input into roll out of new initiatives, and ongoing practice issues-a form of participatory management. Our department level example is termed Faculty Forum and hospital wide example is Provider Advisory Committee (PAC).

- Have anonymous options for any clinician's input that can be processed by a PAC (e.g., surveys, ombudsman representatives to leadership, suggestion boxes that get read, etc.).

- Align patient safety, quality and compliance efforts with clinician wellbeing, keeping in mind that the most conservative workflow decisions are not necessarily the safest.

- Create a culture of accountability of multiple functional sectors of the hospit- 
al operations to improving and sustaining clinician wellbeing.

- Remove barriers for clinicians to obtain help for stress, whether wellness efforts (seminars, coaching, peer support, employee assistance program), or disease management efforts (psychotherapy, medication).

- Organize connections between wellness efforts and reduction of disruptive behavior.

- Respect time off away from work as restorative. Boundaries between work and home need to be preserved as much as possible despite the affordances of technology. Both individual and leader education about this needs to occur to help change the professional and institutional culture.

\section{Acknowledgements}

Alan H. Rosenstein MD, MBA, has presented twice at URMC. His work initially began on disruptive behavior, and later turned to burnout, which helped us understand the relationship between the two and the importance of The Quadruple Aim approach in healthcare. Christine A. Sinsky MD, also presented at URMC. Her work on improving the Joy in the practice of medicine, which also increased satisfaction, revenue, quality and decreased costs was highly instrumental to catalyze an adoption of the Quadruple Aim at URMC. Franziska Plessow PhD for her research and education on how our brains think under stress and the specific dangers involved. Her mentoring in this area helped us gain traction and alignment in making needed changes, given the risks of unaddressed acute and chronic stress. Additionally I would like to thank the multiple faculty and staff, existing wellness initiatives through Human Resources, Employee Assistance Program, and other internal and external partners who collaborated and volunteered their efforts on moving this project forward. Also, thank you to URMC senior administrators and leaders for their forward thinking, compassion, and action in improving the wellbeing of our clinicians, staff and patients.

\section{References}

[1] Shanafelt, T.D., Hasan, O., et al. (2015) Changes in Burnout and Satisfaction With Work-Life Balance in Physicians and the General US Working Population Between 2011 and 2014. Mayo Clinic Proceedings, 90, 1600-1613. https://doi.org/10.1016/j.mayocp.2015.08.023

[2] Epstein, R.M., Privitera, M.R. (2016) Doing Something about Burnout. Lancet, 5, 388, 2216-2217. https://doi.org/10.1016/S0140-6736(16)31332-0

[3] Privitera, M.R., Rosenstein, A.H., Plessow, F. and LoCastro, T. (2015) Physician Burnout and Occupational Stress: An Inconvenient Truth with Unintended Consequences. Journal of Hospital Administration, 4, 27-35.

[4] Lin, Y.-W. (2013) The Causes, Consequences and Mediating Effects of Job Burnout among Hospital Employees in Taiwan. Journal of Hospital Administration, 2, 15-27.

[5] Lyndon, A. (2016) Burnout among Health Professionals and Effect on Patient Safety. AHRQ PS Net. Perspectives on Safety.

https://psnet.ahrq.gov/perspectives/perspective/190/burnout-among-health-professi onals-and-its-effect-on-patient-safety 
[6] ECRI (2017) Burnout in Healthcare Workers: The Elephant in the Room. http://hatrustfunds.com/wp-content/uploads/2017/01/Burnout-in-Healthcare.pdf

[7] Rosenstein, A. and O’Daniel, M. (2017) A Survey of the Impact of Disruptive Behaviors and Communication Defects on Patient Safety. The Joint Commission Journal on Quality and Patient Safety, 34, 464-471. https://doi.org/10.1016/S1553-7250(08)34058-6

[8] Salyers, M., Bonfils, K., Luther. L., Firmin, R., et al. (2017) The Relationship between Professional Burnout and Quality and Safety in Healthcare: A Meta-Analysis. Journal of General Internal Medicine, 32, 475-482. https://doi.org/10.1007/s11606-016-3886-9

[9] DeVoe, J., Fryer, G.E., et al. (2007) Congruent Satisfaction: Is There Geographic Correlation between Patient and Physician Satisfaction? Medical Care, 45, 88-94. https://doi.org/10.1097/01.mlr.0000241048.85215.8b

[10] Friedman, T. (2016) Thank You for Being Late: An Optimists Guide to Thriving in and Age of Accelerations. Farrar, Straus \& Giroux, New York.

[11] Berwick, D.M., Nolan, T.W. and Whittington, J. (2008) The Triple Aim: Care, Health and Cost. Health Affairs, 27, 759-769. https://doi.org/10.1377/hlthaff.27.3.759

[12] Spinelli, W.M. (2013) The Phantom Limb of the Triple Aim. Mayo Clinic Proceedings, 88, 1356-1357. https://doi.org/10.1016/j.mayocp.2013.08.017

[13] Bodenheimer, T. and Sinsky, C. (2014) From Triple to Quadruple Aim: Care of the Patient Requires Care of the Provider. Annals of Family Medicine, 12, 573-576. https://doi.org/10.1370/afm.1713

[14] Sikka, R., Morath, J.M. and Leape, L. (2015) The Quadruple Aim: Care, Health Cost and Meaning in Work. BMJ Quality \& Safety, 24, 1-3. https://doi.org/10.1136/bmjqs-2015-004160

[15] Carayon, P. and Wood, K.E. (2010) Patient Safety: The Role of Human Factors and Systems Engineering. Studies in Health Technology and Informatics, 153, 23-46.

[16] Karwowski, W. (2005) Ergonomics and Human Factors: The Paradigms for Science, Engineering, Design, Technology and Management of Human-Compatible Systems. Ergonomics, 48, 436-463. https://doi.org/10.1080/00140130400029167

[17] Muraven, M. and Baumeister, R.F. (2000) Self-Regulation and Depletion of Limited Resources: Does Self Control Resemble a Muscle? Psychological Bulletin, 126, 247-259. https://doi.org/10.1037/0033-2909.126.2.247

[18] Reason, J. (2005) Safety in the Operating Theatre-Part 2: Human Error and Organizational Failure. Quality \& Safety in Health Care, 14, 50-60.

[19] Duthie, E.A. (2010) Application of Human Error Theory in Case Analysis of Wrong Procedures. Journal of Patient Safety, 6, 108-114. https://doi.org/10.1097/PTS.0b013e3181de47f9

[20] Plessow, F., Fischer, R., Kirschbaum, C. and Goschke, T. (2011) Inflexibility Focused under Stress: Acute Psychosocial Stress Increases Shielding of Action Goals at the Expense of Reduced Cognitive Flexibility with Increasing Time Lag to Stressor. Journal of Cognitive Neuroscience, 23, 3218-3227. https://doi.org/10.1162/jocn_a_00024

[21] Plessow, F., Schade, S., Kirschbaum, C. and Fischer, R. (2012) Better Not to Deal with Two Tasks at the Same Time When Stressed? Acute Psychosocial Stress Reduces Task Shielding in Dual-Task Performance. Cognitive, Affective and Behavioral Neuroscience, 12, 557-570. https://doi.org/10.3758/s13415-012-0098-6 
[22] Savic, I. (2015) Structural Changes of the Brain in Relation to Occupational Stress. Cerebral Cortex, 25, 1554-1564. https://doi.org/10.1093/cercor/bht348

[23] Blix, E., Perski, A., Berglund, H. and Savic, I. (2013) Long-Term Occupational Stress Is Associated with Regional Reductions in Brain Tissue Volumes. PLoS ONE, 8, e64065. https://doi.org/10.1371/journal.pone.0064065

[24] Deligkaris, P., Panagopoulou, E., Montgomery, A.J. and Masoura, E. (2014) Job Burnout and Cognitive Functioning: A Systematic Review. Work \& Stress, 28, 107-123.

[25] Reason, J. (2008) The Human Contribution. Ashgate Publishing Company, Burlington.

[26] Sinsky, C.A. and Privitera, M.R. (2018) Creating a "Manageable Cockpit" for Clinicians: A Shared Responsibility. JAMA Internal Medicine. https://doi.org/10.1001/jamainternmed.2018.0575

[27] Sweller, J. (1988) Cognitive Load during Problem Solving: Effects on Learning. Cognitive Science, 12, 257-285. https://doi.org/10.1207/s15516709cog1202_4

[28] van Merrieboer, J.J.G. and Sweller, J. (2010) Cognitive Load Theory in Health Professional Education: Design Principles and Strategies. Medical Education, 44, 85-93. https://doi.org/10.1111/j.1365-2923.2009.03498.x

[29] West, C.P., Dyrbye, L.N., Erwin, P.J. and Shanafelt, T.D. (2016) Interventions to Prevent and Reduce Physician Burnout: A Systematic Review and Meta-Analysis. The Lancet, 388, 2272-2281. https://doi.org/10.1016/S0140-6736(16)31279-X

[30] Panagioti, M., Panagopoulou, E., Bower, P., et al. (2016) Controlled Interventions to Reduce Burnout in Physicians. A Systematic Review and Meta-Analysis. JAMA Internal Medicine, 177, 195-205. https://doi.org/10.1001/jamainternmed.2016.7674

[31] West, C.P., Dyrbye, L.N., Sloan, J.A. and Shanafelt, T.D. (2009) Single Item Measures of Emotional Exhaustion and Depersonalization Are Useful for Assessing Burnout in Medical Professionals. Journal of General Internal Medicine, 24, 1318-1321. https://doi.org/10.1007/s11606-009-1129-Z

[32] Mind Garden, Inc. http://www.mindgarden.com

[33] de Beer, L., et al. (2013) Linking Employee Burnout to Medical Aid Provider Expenditure. South African Medical Journal, 103, 89-93.

https://doi.org/10.7196/SAMJ.6060

[34] Anagnostopoulous, F., et al. (2012) Physician Burnout and Patient Satisfaction with Consultation in Primary Health Care Settings: Evidence of Relationships from a One-with-Many Design. Journal of Clinical Psychology in Medical Settings, 19, 401-410. https://doi.org/10.1007/s10880-011-9278-8

[35] Michel, A. (2016) Burnout and the Brain. Association for Psychological Science. http://www.psychologicalscience.org/index.php/publications/observer/2016/februar y-16/burnout-and-the-brain.html

[36] Alkadhi, K. (2013) Brain Physiology and Pathophysiology under Mental Stress. ISRN Physiology, 2013, Article ID: 806104. https://doi.org/10.1155/2013/806104

[37] Golkar, A., et al. (2014) The Influence of Work Related Chronic Stress on the Regulation of Emotion and Functional Connectivity in the Brain. PLOS ONE, 9, e104550. https://doi.org/10.1371/journal.pone.0104550

[38] Erickson, S.M., et al. (2017) Putting Patients First by Reducing Administrative Tasks in Healthcare. Position Paper of American College of Physicians. Annals of Internal Medicine, 166, 659-661. https://doi.org/10.7326/M16-2697

[39] Berwick, D.M., et al. (2017) Breaking the Rules for Better Care. JAMA, 317, 
2161-2162. https://doi.org/10.1001/jama.2017.4703

[40] Merlino, J. (2015) The Responsibility Matrix: A Strategy for Stronger Physician/Administrator Partnerships.

http://www.beckershospitalreview.com/hospital-physician-relationships/the-respon sibility-matrix-a-strategy-for-stronger-physician-administrator-partnerships.html

[41] Disconnects in Transforming Health Care Delivery. How Executives, Clinical Leaders, and Clinicians Must Bridge Their Divide and Move forward Together. https://catalyst.nejm.org/

[42] Shanafelt, T.D., Dyrbye, L.N. and West, C.P. (2017) Addressing Physician Burnout. The Way Forward. JAMA, 317, 901-902. https://doi.org/10.1001/jama.2017.0076

[43] Wallace, J.E., Lemaire, J.B. and Ghali, W.A. (2009) Physician Wellness: A Missing Quality Indicator. The Lancet, 374, 1714-1721.

https://doi.org/10.1016/S0140-6736(09)61424-0 